\title{
Comparative evaluation of performance of percolated perpendicular media using FePt-MgO/Pt/Cr trilayer and FePt-MgO/MgO bilayer
}

\author{
A.C. Sun ${ }^{\text {a }}$, Y.C. Tsai ${ }^{\text {b }}$, Jen-Hwa Hsu ${ }^{\text {a,* }}$, P.C. Kuo ${ }^{\text {b }}$, H.L. Huang ${ }^{\text {a }}$ \\ a Department of Physics and Center for Nanostorage Research, National Taiwan University, Taipei 106, Taiwan \\ ${ }^{\mathrm{b}}$ Departments of Materials Science and Engineering, National Taiwan University, Taipei 106, Taiwan
}

\section{A R T I C L E I N F O}

Available online 8 August 2008

PACS:

75.50.Ss

75.50.Bb

75.50.Vv

74.25. Ha

75.60.-d

Keywords:

FePt

Percolated perpendicular media

Perpendicular magnetic recording

Underlayer

\begin{abstract}
A B S T R A C T
$\left(\mathrm{Fe}_{48} \mathrm{Pt}_{52}\right)_{100-x}-(\mathrm{MgO})_{x}$ films were used to examine the performance of a perpendicular percolated medium. Two underlayers, $\mathrm{Pt}(001) / \mathrm{Cr}(002)$ and $\mathrm{MgO}(002)$, were used for comparison. The $\left(\mathrm{Fe}_{48} \mathrm{Pt}_{52}\right)_{100-x}-(\mathrm{MgO})_{x}$ film with the $\mathrm{MgO}$ underlayer exhibits a strong preference to segregate at FePt grain boundaries. The microstructure with small closely packed MgO particles (2-4 nm) dispersed uniformly in the $\mathrm{L} 1_{0}$ FePt matrix was achieved in the $\mathrm{Pt} / \mathrm{Cr}$ underlayered sample. Structural data reveal that the precipitate is crystallographically coherent with the surrounding $\mathrm{L} 1_{0} \mathrm{FePt}$ phase and preserves good lattice alignment. Magnetic results indicate significant pinning behavior for those introduced nonmagnetic columns with an enhanced coercivity of about 70\%-much greater than that of the MgO underlayered samples. Percolated perpendicular medium can be realized in the FePt system and a $\mathrm{Pt}\left(\begin{array}{l}0 \\ 0\end{array}\right) / \mathrm{Cr}\left(\begin{array}{l}0 \\ 0\end{array}\right)$ underlayer promotes the formation of pinning sites within the FePt grains.
\end{abstract}

(c) 2008 Elsevier B.V. All rights reserved.

\section{Introduction}

Perpendicular magnetic recording (PMR), presently used in hard disk drives (HDD), began to replace traditional longitudinal magnetic recording (LMR) in 2005 [1]. It has several advantages, such as a sharper gap field, a more stable magnetic configuration, a smaller demagnetization field and others. Currently CoCrPtbased material such as $\mathrm{CoCrPt}-\mathrm{SiO}_{2}$ is used in the PMR medium with a granular structure $[2,3]$. The so-called granular perpendicular medium (GPM) has been successfully demonstrated to reach a recording density of $345 \mathrm{~Gb} / \mathrm{in}^{2}$ in 2006 [4]. The decoupled magnetic grains of reduced size markedly enhance the recording resolution and the recording density.

However, when the recording density reaches $500 \mathrm{~Gb} / \mathrm{in}^{2}$, the superparamagnetic effect will be encountered and must be overcome [3]. Transition jitter noise $[5,6]$ becomes a serious problem because grain size reduction is limited. A percolated perpendicular medium (PPM) has been proposed to increase the resolution of the signal without sacrificing thermal stability [7]. The PPM comprises crystallographically oriented, closely packed and interconnected magnetic grains with strong perpendicular anisotropy. The densely packed non-magnetic particles with nanometer sizes, which act as pinning sites, are uniformly

\footnotetext{
* Corresponding author. Tel.: +88623366 5162; fax: +886233665892.

E-mail address: jhhsu@phys.ntu.edu.tw (J.-H. Hsu).
}

distributed in the magnetic matrix. In PPM, the transient region that is essentially determined from the distribution of densely dispersed pinning sites may be much smoother than that in GPM, so the jitter noise can be substantially lower. Meanwhile, ferromagnetic exchange coupling between adjacent magnetic grains improves thermal stability without resulting in high coercivity-another advantage in recording applications. Since the PPM idea has been proposed, attempts to realize PPM have been made in Co-based films using different approaches, such as those of Laughlin et al. [8,9] and Rahman et al. [10,11]. However, efforts in FePt, an extremely promising candidate for ultra-high density recording, are still lacking.

In our earlier work, a highly magnetic anisotropic FePt film with an $\mathrm{L} 1_{0}$ phase at low deposition temperature was prepared without any post-annealing treatment [12]. Therefore, in this study, MgO was used as the pinning site material to evaluate the PPM behavior in the $\mathrm{FePt}\left(\begin{array}{lll}0 & 0 & 1\end{array}\right) / \mathrm{Pt}\left(\begin{array}{lll}0 & 0 & 1\end{array}\right) / \mathrm{Cr}\left(\begin{array}{lll}0 & 0 & 2\end{array}\right)$ trilayer. $\mathrm{MgO}$ is selected for the following reasons: (1) it is nearly insoluble in FePt, and (2) it induces and maintains the $(001)$ orientation of the $\mathrm{Ll}_{0}$ phase [13]. $\mathrm{MgO}(002)$ films have frequently been used as underlayers in growing the $\mathrm{Ll}_{0}$ FePt phase [14]. Accordingly, PPM performance associated with $\mathrm{FePt}(001) / \mathrm{MgO}(002)$ was also studied and the results compared with those from $\mathrm{FePt}(001) /$ $\mathrm{Pt}(001) / \mathrm{Cr}(002)$. A clue for preparing the medium with superior PPM behavior and optimum microstructure at low process temperature can be obtained, enabling the recording density to be increased above $500 \mathrm{~Gb} / \mathrm{in}^{2}$ in the near future. 


\section{Experiments}

Since FePt magnetic layer on different underlayers, $\mathrm{Pt}\left(\begin{array}{l}0 \\ 0\end{array} 1\right)$ and $\mathrm{MgO}(002)$ exhibits extensive ordering at different substrate temperatures [12,15], the deposition temperatures of the Pt and $\mathrm{MgO}$ underlayered samples were set to 420 and $580^{\circ} \mathrm{C}$, respectively. Composite $(\mathrm{FePt})_{100-x}-(\mathrm{MgO})_{x}$ films $(x=0-6.13 \mathrm{vol} \%)$ were magnetron-sputtered with a background vacuum of under $6.7 \times 10^{-7} \mathrm{~Pa}$. Before the FePt-MgO film was deposited, $\mathrm{Pt}(001) /$ $\mathrm{Cr}\left(\begin{array}{lll}0 & 0 & 2\end{array}\right)$ and $\mathrm{MgO}\left(\begin{array}{lll}0 & 02\end{array}\right)$ underlayers were deposited at $350^{\circ} \mathrm{C}$ and room temperature, respectively. The thicknesses of the FePt-MgO layer were fixed at $20 \mathrm{~nm}$, and the Pt and $\mathrm{MgO}$ layers were set to 3 and $10 \mathrm{~nm}$. Various rf sputtering powers of $\mathrm{MgO}$ were used to control the volume fraction of $\mathrm{MgO}$ in composite FePt-MgO film. The crystalline phases and microstructures of the films were identified by X-ray diffraction (XRD) using $\mathrm{Cu}-\mathrm{K}_{\alpha}$ radiation and high-resolution transmission electron microscopy (HRTEM), respectively. The chemical composition of the magnetic FePt layer was determined by energy dispersive spectroscopy (EDS), which revealed that the Pt content in the FePt film was 52 at\%. Since $\mathrm{MgO}$ is almost immiscible with the magnetic FePt phase, the $\mathrm{MgO}$ content of the magnetic FePt matrix was determined using the formula $\left[R_{\mathrm{MgO}} /\left(R_{\mathrm{FePt}}+R_{\mathrm{MgO}}\right)\right] \times 100 \mathrm{vol} \%$, where $R$ denotes the sputtering rate. Magnetic properties were examined using a vibrating sample magnetometer (VSM) and a superconducting quantum interference device magnetometer (SQUID) at room temperature with maximum applied fields of 1100 and $4800 \mathrm{kA} / \mathrm{m}$, respectively.

\section{Results and discussions}

Fig. 1 presents the microstructural evolution of the two series of $\left(\mathrm{Fe}_{48} \mathrm{Pt}_{52}\right)_{100-x}-(\mathrm{MgO})_{x}$ films with $x$ from 0 to $6.13 \mathrm{vol} \%$. The mean grain size was about $60 \mathrm{~nm}$ in the pure FePt film with the Pt underlayer. At $x=0.08$, no significant $\mathrm{MgO}$ precipitate was observed and the grain size remained unchanged. In the sample with $x=0.15$, the densely segregated particulate $\mathrm{MgO}$ of a uniform size of $2-4 \mathrm{~nm}$ was dispersed evenly in the FePt matrix as shown in Fig. 1(c). The composition was identified using nanobeam EDS. Fig. 2 displays the high-resolution image of the spot in Fig. 1(c) marked with dotted circle. It further revealed that the phase boundaries of the precipitates were crystallographically coherent with the FePt phase matrix, which suggests that the segregation process may not influence the lattice orientation of
FePt phase. These microstructure features perfectly fulfill the requirements of PPM. When $x$ was increased to 1.67 , the grain size declined and the precipitated $\mathrm{MgO}$ became irregular in shape and located preferentially at FePt grain boundaries. At $x=6.13, \mathrm{MgO}$ precipitates grew as normal grains with a wide size distribution, and joined FePt grains, as presented in Fig. 1(e). The FePt-MgO films deposited on the MgO layer exhibited significantly different segregation behavior. The initial grain size (at $x=0$ ) resembled that with a Pt underlayer, even when deposited at a higher substrate temperature. Segregation of $\mathrm{MgO}$ began at $x=0.15$. The precipitated particles appeared preferentially at the grain boundaries of FePt and exhibited little uniformity in both size and dispersion; this fact is associated with the higher deposition temperature. In the film with $x=0.76$ [Fig. 1(h)], small irregularly shaped $\mathrm{MgO}$ particles appeared at FePt grain boundary and partially separated the FePt grains, as in the Pt-underlayered film with $x=1.67$. As $x$ was increased to $1.23, \mathrm{MgO}$ completely surrounded the FePt grains, forming a well-defined granular structure as the size decreased. When $x=2.61$, the microstructure

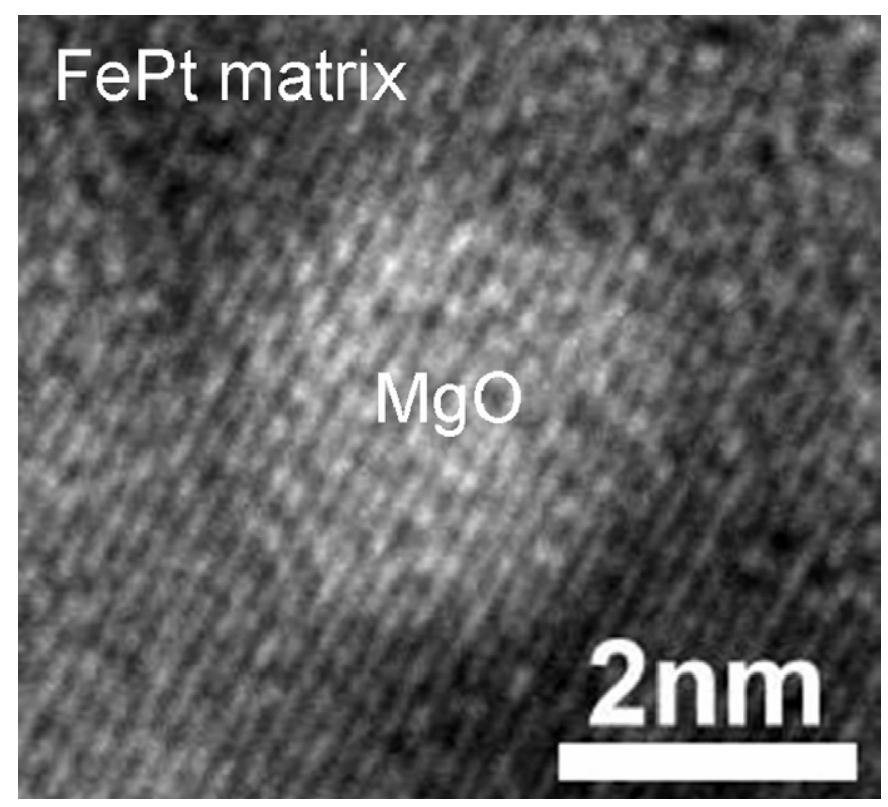

Fig. 2. High-resolution transmission electron image of MgO nano-particle and its surroundings of $\mathrm{L} 1_{0}$ FePt matrix.
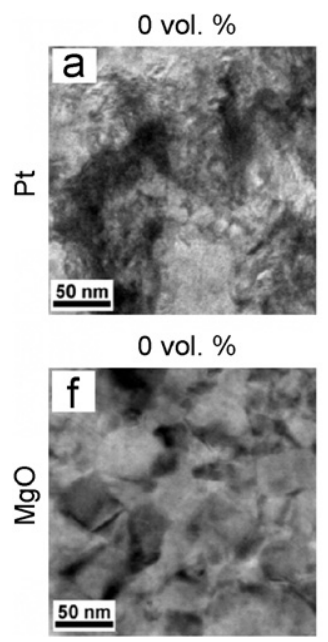

0.08 vol. $\%$

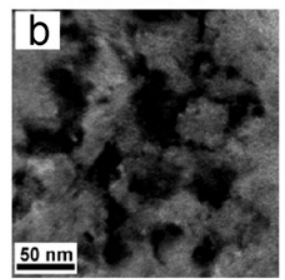

0.15 vol. $\%$

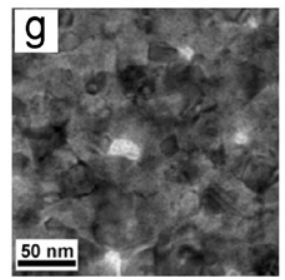

0.15 vol. $\%$

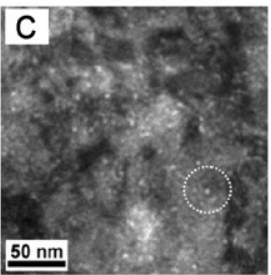

0.76 vol. \%

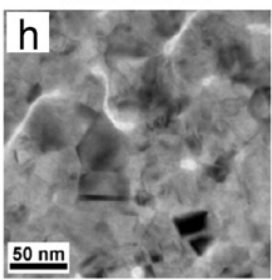

1.67 vol. \%

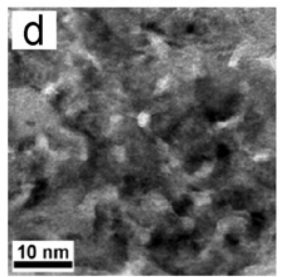

1.23 vol. $\%$

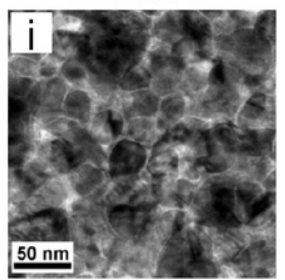

6.13 vol. $\%$

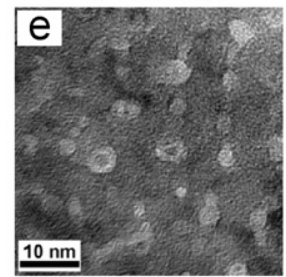

2.61 vol. \%

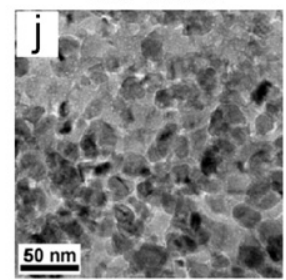

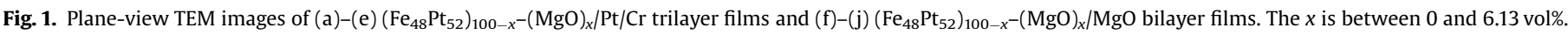


remained granular but the average size of FePt was further reduced and the size distribution became narrower, as presented in Fig. 1(j). The TEM images demonstrate that the Pt underlayer helps the formation of $\mathrm{MgO}$ pinning sites within FePt grains.

The XRD data for the Pt underlayered FePt-MgO films with various $\mathrm{MgO}$ contents show that the initial incorporation of $\mathrm{MgO}$ does not alter the crystalline structure [16] until the amount of $\mathrm{MgO}$ exceeds $0.15 \mathrm{vol} \%$. A transformation of lattice orientation was observed at $x \geqslant 0.15$. The $\mathrm{L} 1_{0}\left(\begin{array}{ll}0 & 02\end{array}\right)$ peak transformed gradually to the $(200)$ peak as $x$ was increased, revealing that the magnetic easy-axis tilted from perpendicular to the in-plane direction. In the $\mathrm{MgO}$ underlayered series, the crystal orientation shows insensitive to the $\mathrm{MgO}$ dopant content [15]. However, the alignment of crystal orientation in the $\mathrm{MgO}$ series is weaker than in the Pt series, as evidenced by the wider FWHM. The FePt (111) peak is found in addition to $\operatorname{FePt}(002)$ peak.

Figs. 3(a) and (b) present cross-sectional TEM images of the films on textured $\operatorname{Pt}(001) / \operatorname{Cr}(002)$ bilayer films to illustrate the layer structure and the location of $\mathrm{MgO}$ defects. No physical separation existed between FePt grains (Fig. 1(a)), indicating that a continuous film structure exists in pure FePt films. Lateral grain size was roughly $50-60 \mathrm{~nm}$. The cross section shows $\mathrm{Cr}$ grains in the $\mathrm{Cr}$ underlayer as columns grown perpendicular to the films plane due to expitaxial growth starting from the $\mathrm{Cr}$ underlayer and continuing through the Pt intermediate layer and extending into the FePt layer as described by An-Cheng Sun et al. [17,18]. The fct FePt grains retains highly textured crystallographically, with the $c$-axis perpendicular to the film plane. Additionally, a very flat surface (roughness $<1 \mathrm{~nm}$ ) was acquired for pure FePt film. At $x=0.15$, the lateral grain size of magnetic layer was about the same as that in pure FePt film but with a significantly rougher surface. This rough surface is artificial and arises from sample damage during TEM specimen preparation. The above statement can be argued from this way that the same roughness appears at the $\mathrm{FePt} / \mathrm{Pt}$ and $\mathrm{Pt} / \mathrm{Cr}$ interfaces as observed in the right side of Fig. 3(b). Another difference at $x=0.15$ is that some small lightgrey columns marked by the white arrows in Fig. 3(b), $20 \mathrm{~nm}$ high and $4 \mathrm{~nm}$ wide, intercalate between large columnar grains. Determined by nanobeam EDS, the small gray columns are predominantly MgO. For comparison, Figs. 3(c) and (d) present cross-sectional TEM images of $(\mathrm{FePt})_{100-x}-(\mathrm{MgO})_{x} / \mathrm{MgO}(002)$ bilayer films with $x=0$ and 1.23 , respectively. The high-resolution cross-sectional TEM images demonstrate that the $\operatorname{FePt}(001)$ grains were also epitaxially grown on the $\mathrm{MgO}(002)$ underlayer (data not shown) in these two samples. The grains with a lateral size of $40-60 \mathrm{~nm}$ were well connected in the pure FePt layer (Fig. 3(c)). The surface roughness of the pure FePt film was approximately $5 \mathrm{~nm}$, which is larger than that in the $\mathrm{FePt} / \mathrm{Pt} / \mathrm{Cr}$ trilayer film due to high deposition temperature. After adding $\mathrm{MgO}$ of $1.23 \mathrm{vol} \%$ to the FePt film, the FePt-MgO layer had some isolated FePt grains separated by clear MgO boundaries (white arrows in Fig. 3(d)) that have similar column-like shapes. The MgO boundary can impede grain growth of the FePt phase, thereby reducing lateral grain size of FePt to about $30 \mathrm{~nm}$. Surface roughness was increased to about $15 \mathrm{~nm}$ in the $(\mathrm{FePt})_{98.77}-(\mathrm{MgO})_{1.23}$ film. Although the FePt films with different underlayers have similar columnar structures of incorporated $\mathrm{MgO}, \mathrm{MgO}$ typically precipitates within the FePt grain in $\mathrm{Pt}$ underlayered film and segregates at the FePt grain boundary in $\mathrm{MgO}$ underlayered film as indicated by the plane-view TEM images. To further locate the MgO columns in FePt film, the contrast of the $\mathrm{MgO}$ column with neighboring $\mathrm{FePt}$ grains indicates that for precipitation of $\mathrm{MgO}$ within the FePt grain, the $\mathrm{MgO}$ column was embedded in the FePt matrix and surrounded by FePt grains (Fig. 2). Since the electron beam is a finite-size spot, the contrast of $\mathrm{MgO}$ column size with neighboring FePt grains is reduced due to mixed images of $\mathrm{MgO}$ and $\mathrm{FePt}$ and exhibits blurred FePt/MgO interfaces. Rather, the MgO columns segregated at the grain boundary were also surrounded by $\mathrm{MgO}$, generating increased contrast sharpness [19]. The images of boundaries separated by FePt and $\mathrm{MgO}$ are very distinct. For the PPM requirement, columnar oxide pinning sites have advantages over oxide dots [9]. This advantage is because columnar oxide leads to stronger pinning effect on domain wall motion. The columnar $\mathrm{MgO}$ structure has been observed in both $(\mathrm{FePt})_{99.85}-(\mathrm{MgO})_{0.15} /$ $\mathrm{Pt} / \mathrm{Cr}$ trilayer and $(\mathrm{FePt})_{98.77}-(\mathrm{MgO})_{1.23} / \mathrm{MgO}$ bilayer films, meeting the PPM requirements. However, precipitation within the FePt grain and segregation at the FePt boundary result in different influences on magnetic properties and magnetization reversal behaviors, which are discussed later.

Fig. 4(a) plot the dependence of the perpendicular coercivity $\left(H_{\mathrm{c} \perp}\right)$ on the $\mathrm{MgO}$ dopant content of the Pt and $\mathrm{MgO}$ underlayered $\left(\mathrm{Fe}_{48} \mathrm{Pt}_{52}\right)_{100-x}-(\mathrm{MgO})_{x}$ samples, respectively. In the Pt series, when $0.15 \mathrm{vol} \% \mathrm{MgO}$ is added, $H_{\mathrm{c} \perp}$ increased rapidly from $167 \mathrm{kA} / \mathrm{m}$ to a maximum value of $287 \mathrm{kA} / \mathrm{m}$. As $x$ increased further, $H_{\mathrm{c} \perp}$ declined drastically to $127 \mathrm{kA} / \mathrm{m}(x=6.13) . H_{\mathrm{c} \perp}$ can be increased substantially by $70 \%$ with the Pt underlayer. Films with an $\mathrm{MgO}$ underlayer saw only a $23 \%$ increase in $H_{\mathrm{c} \perp \text {, even though }}$ $H_{\mathrm{c} \perp}$ peaks at a higher MgO content, as shown in Fig. 4(a). The enhancement of $H_{\mathrm{c} \perp}$ has several causes. (1) The ordered FePt phase has a very large anisotropy constant $\left(K_{\mathrm{u}}\right)$, and increasing ordering parameter can increase the coercivity. (2) Reducing the magnetic grain size may increase the coercivity when the size is close to the single domain size [20]. (3) Isolating the magnetic grain can reduce the intergrain exchange coupling, increasing coercivity [21]. (4) The MgO particles hinder wall motion, providing the extra pinning sites that increase the energy barrier to the propagation of the domain wall. The above results in $\left(\mathrm{Fe}_{48} \mathrm{Pt}_{52}\right)_{100-x}-(\mathrm{MgO})_{x} / \mathrm{Pt} / \mathrm{Cr}$ trilayer films yield a similar microstructure (grain size, grain size distribution, and grain to grain interconnection) for $x=0$ and 0.15 . The value of $S_{\text {order }}$ remains unchanged in the range $0.7-0.75$ as $x$ was increased from 0 to 0.15 [Fig. 4(b)], and the crystal alignment appeared to be similar.
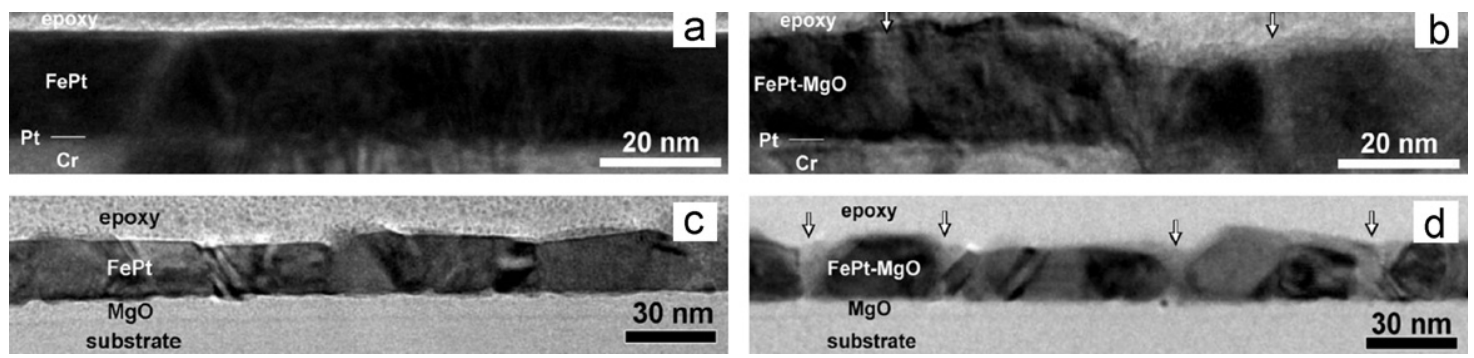

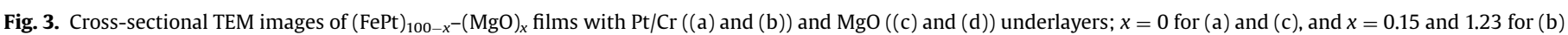
and (d), respectively. 


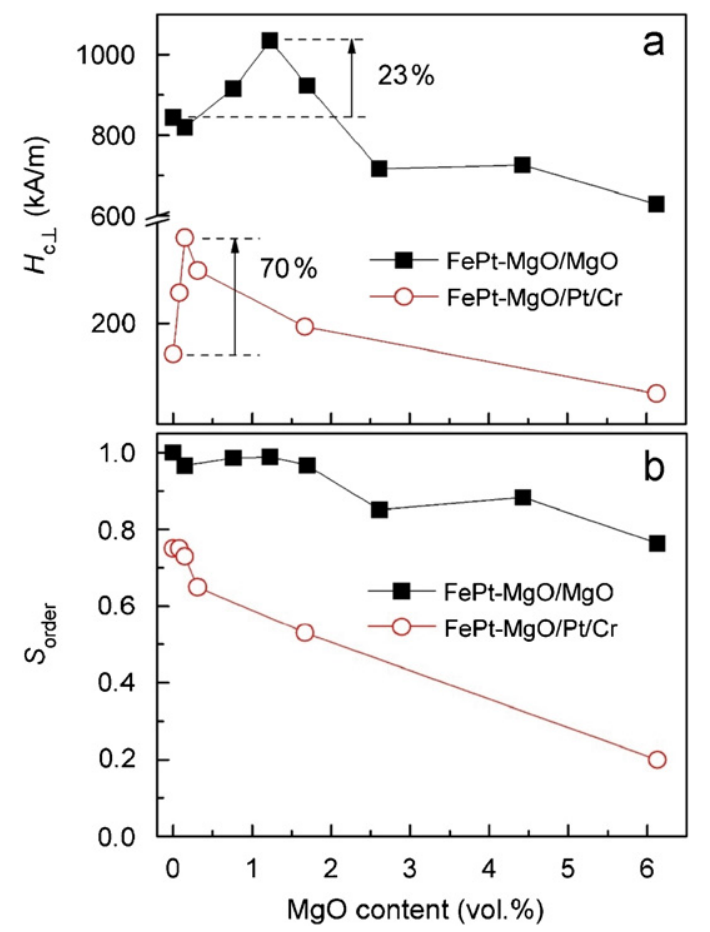

Fig. 4. Dependence of the (a) perpendicular coercivity $\left(H_{\mathrm{c} \perp}\right)$ and (b) ordering parameters on the $\mathrm{MgO}$ dopant content of the $\mathrm{Pt}$ and $\mathrm{MgO}$ underlayered $\left(\mathrm{Fe}_{48} \mathrm{Pt}_{52}\right)_{100-x}-(\mathrm{MgO})_{x}$ samples, respectively.

Hence, the ordering effect, magnetic grain size, and grain size distribution were not the main causes of the enhanced coercivity. The only difference between $x=0$ and 0.15 is the appearance of $\mathrm{MgO}$ within FePt grain. The MgO particles of size $2-4 \mathrm{~nm}$ are comparable to the domain wall width of the $\mathrm{L} 1_{0}$ FePt phase [22]. Accordingly, the particles serve as effective pinning sites. The microstress that is caused by the lattice mismatch at the $\mathrm{FePt}\left(\begin{array}{lll}0 & 0 & 1\end{array}\right) / \mathrm{MgO}\left(\begin{array}{ll}0 & 02\end{array}\right)$ interface, which is roughly $9 \%$ [17], is also responsible for the pinning. Therefore, an $H_{\mathrm{c} \perp}$ enhancement of about $70 \%$ is associated mainly with the precipitation of $\mathrm{MgO}$ particles in the FePt matrix. As $x$ was increased from 0.15 to 6.13, the decline in ordering reduced $H_{\mathrm{c} \perp}$.

Angular dependence of coercivity for FePt- $\mathrm{MgO}_{0.15} / \mathrm{Pt} / \mathrm{Cr}$ and FePt- $\mathrm{MgO}_{1.23} / \mathrm{MgO}$ films were measured to further investigate the difference in magnetic reversal mode. These two samples give the largest enhancement of $H_{\mathrm{c} \perp}$ in two series, but exhibit different microstructure of typical percolated and granular type respectively as shown in Fig. 1 . The coercivities at $0^{\circ}$ and $90^{\circ}$ represent the applied field vertical and parallel to the film plane, respectively. For the reversal process from domain wall motion, the coercivity at $\theta$ should be proportional to $H / \cos (\theta)$, where $\theta$ is the angle between the applied field and the easy axis of the uniaxial anisotropy and $H_{\mathrm{c}}(+)$ is the coercivity when the direction of applied field is parallel to the easy axis. The ideal $\theta$ dependence of coercivity for the rotation mechanism derived from the StonerWohlfarth model was also plotted for comparison. The results shown in Fig. 5 indicate that the rotation reversal is predominant in the granular sample; on the contrary, pinning behavior is more apparent in the percolated one. In the granular-type film, the separation of magnetic phase with single domain size constrains the continuous motion of reversal domain walls. Independent nucleation of reversal domain in each isolated grain thus becomes favorable, which leads the angular dependence of coercivity toward the Stoner-Wohlfarth behavior. On the other hand,

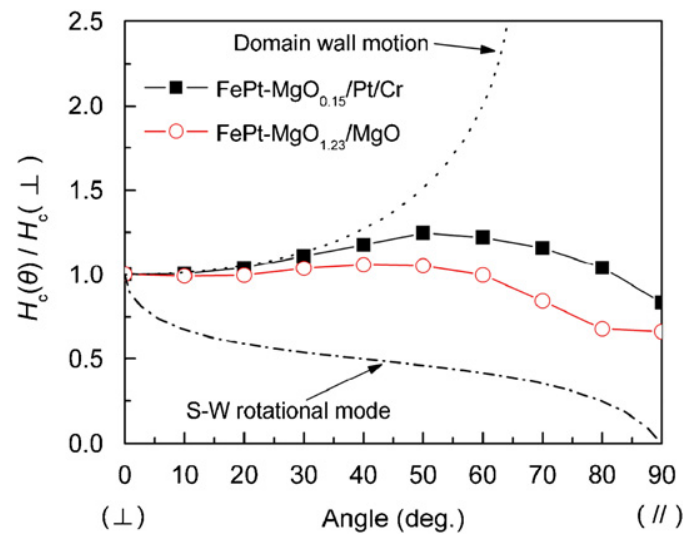

Fig. 5. Angular dependence of coercivity for the samples of $\mathrm{FePt}-\mathrm{MgO}_{0.15} / \mathrm{Pt} / \mathrm{Cr}$ and $\mathrm{FePt}-\mathrm{MgO}_{1.23} / \mathrm{MgO}$.

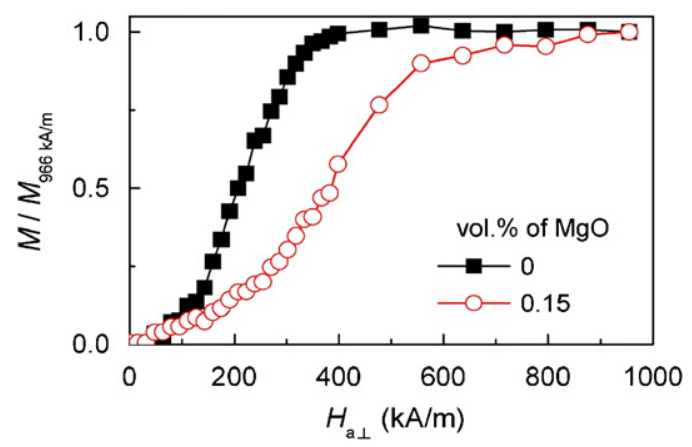

Fig. 6. The initial magnetization curves of $\mathrm{FePt} / \mathrm{Pt} / \mathrm{Cr}$ and $(\mathrm{FePt})_{99.85}-(\mathrm{MgO})_{0.15} / \mathrm{Pt} /$ Cr trilayer films.

although the domain wall motion was impeded by MgO dots in the percolated sample, the magnetic phase still remains interconnected. Therefore, similar to the pure FePt sample, the magnetic reversal of $\mathrm{FePt}-\mathrm{MgO}_{0.15} / \mathrm{Pt} / \mathrm{Cr}$ film is mainly dominated by domain-wall-motion mechanism.

Fig. 6 plots the initial magnetization curves of an FePt film and an $(\mathrm{FePt})_{99.85}-(\mathrm{MgO})_{0.15}$ film to elucidate the relationship between the $\mathrm{MgO}$ pinning sites and the magnetization behavior. The FePt film exhibits strong domain wall motion. When $\mathrm{MgO}$ is incorporated into the FePt matrix, domain wall pinning dominates the initial magnetization state [23], suggesting that $\mathrm{MgO}$ strengthens the wall pinning effect. The pinning effect in the FePt film is weak. Therefore, a rapid increase in the magnetization occurs at low applied fields. When $\mathrm{MgO}$ is present in the FePt matrix, the pinning field increases and domain wall motion is retarded.

\section{Conclusion}

$\left(\mathrm{Fe}_{48} \mathrm{Pt}_{52}\right)_{100-x}-(\mathrm{MgO})_{x}$ films with $x$ from 0 to $6.13 \mathrm{vol} \%$ on $\mathrm{Pt}$ and $\mathrm{MgO}$ underlayers were fabricated. A microstructure with close-packed $\mathrm{MgO}$ particles of very small size (2-4 nm), dispersed uniformly in the $\mathrm{L} 1_{0}$ FePt matrix, was formed in the Pt underlayered sample with $x=0.15$. Structural data demonstrate that the precipitate is crystallographically coherent with the surrounding $\mathrm{L} 1_{0}$ FePt phase and retains a good lattice alignment. The coercivity was enhanced substantially by significant pinning behavior of the incorporated non-magnetic defects. The idea of PPM can be realized in a FePt system. 


\section{Acknowledgment}

This work was supported by the Ministry of Economic Affairs of Taiwan.

\section{References}

[1] M. Xiao, H. Do, W. Weresin, Q. Dai, Y. Ikeda, K. Takano, A. Moser, B. Lengsfield A. Berger, M. Schabes, V. Nayak, N. Supper, H. Rosen, B. Marchon, M. Minardi, B. Heinz, J. Appl. Phys. 99 (2006) 08E712.

[2] J.D. Dutson, M. Hashimoto, Y. Inaba, S.J. Greaves, H. Muraoka, K. O’Grady, IEEE Trans. Magn. 43 (2007) 814.

[3] S.N. Piramanayagam, J.Z. Shi, H.B. Zhao, C.K. Pocl, C.S. Mah, C.Y. Ong, J.M. Zhao J. Zhang, Y.S. Kay, L. Lu, IEEE Trans. Magn. 43 (2007) 633.

[4] 〈http://www.hitachigst.com/hdd/research/recording_head/pr/index.html 〉.

[5] A. Takeo, Y. Takahashi, Y. Tanaka, K. Miura, H. Muraoka, Y.H. Nakamura, J. Appl Phys. 87 (2000) 4987.

[6] K. Miura, H. Muraoka, H. Aoi, Y. Nakamura, J. Magn. Magn. Mater. 287 (2005) 133

[7] Jian-Gang Zhu, Yuhui Tang, J. Appl. Phys. 99 (2006) 08 Q903.

[8] David Laughlin, Yingguo Peng, Yue-Ling Qin, Mark Lin, Jian-Gang Zhu, IEEE Trans. Magn. 43 (2007) 693.

[9] Yue-Ling Qin, David Laughlin, Yingguo Peng, Jian-Gang Zhu, IEEE Trans. Magn. 43 (2007) 2136
[10] M. Tofizur Rahman, Nazmun Shams, Yun-Chung Wu, Chih-Huang Lai, Appl. Phys. Lett. 91 (2007) 132505.

[11] M. Tofizur Rahman, Chih-Huang Lai, D. Vokoun, Nazmun Shams, IEEE Trans. Magn. 43 (2007) 2133.

[12] An-Cheng Sun, Jen-Hwa Hsu, P.C. Kuo, H.L. Huang, Thin Solid Films 516 (2008) 1155

[13] T. Suzuki, T. Kiya, N. Honda, K. Ouchi, IEEE Trans. Magn. 36 (2000) 2417.

[14] Z.L. Zhao, J.S. Chen, J. Ding, J.B. Yi, B.H. Liu, J.P. Wang, Appl. Phys. Lett. 88 (2006) 052503.

[15] An-Cheng Sun, Y.C. Tsai, Jen-Hwa Hsu, P.C. Kuo, H.L. Huang, Puneet Sharma, Phys. Status Solidi (c) 4 (2007) 4487.

[16] An-Cheng Sun, Jen-Hwa Hau, P.C. Kuo, H.L. Huang, IEEE Trans. Magn. 43 (2007) 2130.

[17] An-Cheng Sun, P.C. Kuo, Jen-Hwa Hsu, H.L. Huang, Jui Sun, J. Appl. Phys. 98 (2005) 076109

[18] An-Cheng Sun, P.C. Kuo, Jen-Hwa Hsu, H.L. Huang, J. Appl. Phys. 99 (2006) $08 \mathrm{E} 709$.

[19] David B. Williams, C. Barry Carter, Transmission Electron Microscopy, Plenum Press, New York, London, 1996, p. 444.

[20] T. Shima, K. Takanashi, Y.K. Takshashi, K. Hono, Appl. Phys. Lett. 85 (2005) 2571.

[21] J.S. Chen, B.C. Lin, J.F. Hu, B. Liu, G.M. Chow, G. Ju, Appl. Phys. Lett. 91 (2007) 132506.

[22] R. Ploessl, J.N. Chapman, J. Appl. Phys. 74 (1993) 7431.

[23] Y.Z. Zhou, J.S. Chen, G.M. Chow, J.P. Wang, J. Appl. Phys. 95 (2004) 7495. 